\title{
Therapeutic effect of hepatocyte growth factor-secreting mesenchymal stem cells in a rat model of liver fibrosis
}

\author{
Myung-Deok Kim ${ }^{1,2,6}$, Sung-Soo Kim ${ }^{3,4,6}$, Hyun-Young Cha ${ }^{1}$, Seung-Hun Jang ${ }^{1,2}$, Da-Young Chang ${ }^{3}$, \\ Wookhwan Kim ${ }^{2,5}$, Haeyoung Suh-Kim ${ }^{3}$ and Jae-Ho Lee ${ }^{1,2}$
}

Bone marrow-derived mesenchymal stromal cells (MSCs) have been reported to be beneficial for the treatment of liver fibrosis. Here, we investigated the use of genetically engineered MSCs that overexpress hepatocyte growth factor (HGF) as a means to improve their therapeutic effect in liver fibrosis. Liver fibrosis was induced by intraperitoneal injection of dimethylnitrosamine. HGF-secreting MSCs (MSCs/HGF) were prepared by transducing MSCs with an adenovirus carrying HGF-encoding cDNA. MSCs or MSCs/HGF were injected directly into the spleen of fibrotic rats. Tissue fibrosis was assessed by histological analysis 12 days after stem cell injection. Although treatment with MSCs reduced fibrosis, treatment with MSCs/HGF produced a more significant reduction and was associated with elevated HGF levels in the portal vein. Collagen levels in the liver extract were decreased after MSC/HGF therapy, suggesting recovery from fibrosis. Furthermore, liver function was improved in animals receiving MSCs/HGF, indicating that MSC/HGF therapy resulted not only in reduction of liver fibrosis but also in improvement of hepatocyte function. Assessment of cell and biochemical parameters revealed that mRNA levels of the fibrogenic cytokines PDGF-bb and TGF- $\beta 1$ were significantly decreased after MSC/HGF therapy. Subsequent to the decrease in collagen, expression of matrix metalloprotease-9 (MMP-9), MMP-13, MMP-14 and urokinase-type plasminogen activator was augmented following MSC/HGF, whereas tissue inhibitor of metalloprotease-1 (TIMP-1) expression was reduced. In conclusion, therapy with MSCs/ HGF resulted in an improved therapeutic effect compared with MSCs alone, probably because of the anti-fibrotic activity of HGF. Thus, MSC/HGF represents a promising approach toward a cell therapy for liver fibrosis.

Experimental \& Molecular Medicine (2014) 46, e110; doi:10.1038/emm.2014.49; published online 22 August 2014

\section{INTRODUCTION}

Chronic liver damage leads to liver fibrosis, which is characterized by the hyper-accumulation of extracellular matrix $(\mathrm{ECM})$ proteins including collagen, ${ }^{1}$ which produces a fibrous scar that distorts the hepatic architecture and causes liver cell dysfunction. Currently, there is no effective therapy for liver fibrosis, and liver transplantation, which is a major surgery and is limited by an inadequate supply of transplantable livers, is the only option for end-stage liver failure.

Mesenchymal stromal cells (MSCs) were recently found to provide effective therapy in animal models of liver fibrosis and cirrhosis. Sakaida et $a .^{2}$ and Fang et al. ${ }^{3}$ showed that transplantation of bone marrow cells or systemic infusion of FLK1 $(+)$ MSCs, respectively, reduced carbon tetrachloride (CCl4)-induced liver fibrosis in mice. A therapeutic effect of transplanting hepatocyte growth factor (HGF)-treated bone marrow MSCs was also documented in $\mathrm{CCl}_{4}$-injured rats. ${ }^{4}$ MSCs were shown to differentiate into hepatocytes in vitro ${ }^{5-7}$ and in vivo. ${ }^{8}$ Collectively, these results establish a therapeutic effect of MSCs in liver fibrosis. However, the therapeutic efficacy of MSC transplantation was not significant in some reports. Carvalho et al. ${ }^{9}$ reported that bone marrow-derived MSCs were unable to reduce liver

\footnotetext{
${ }^{1}$ Department of Biochemistry, Ajou University School of Medicine, Suwon, Republic of Korea; ${ }^{2}$ Chronic Inflammatory Disease Research Center, Ajou University School of Medicine, Suwon, Republic of Korea; ${ }^{3}$ Department of Anatomy, Ajou University School of Medicine, Suwon, Republic of Korea; ${ }^{4}$ Center for Cell Death Regulating Biodrug, Ajou University School of Medicine, Suwon, Republic of Korea and ${ }^{5}$ Department of General Surgery, Ajou University School of Medicine, Suwon, Republic of Korea

${ }^{6}$ These two authors contributed equally to this work.

Correspondence: Professor H Suh-Kim, Department of Anatomy, Ajou University School of Medicine, Youngtong-gu, Woncheon-dong, San 5, Suwon 443-749, Republic of Korea.

E-mail: hysuh@ajou.ac.kr

or Professor J-H Lee, Department of Biochemistry, Ajou University School of Medicine, Yeongtong-gu, Wonchen-dong, San 5, Suwon 443-721, Republic of Korea. E-mail: jhlee64@ajou.ac.kr

Received 11 May 2014; accepted 8 June 2014
} 
fibrosis or improve liver function in a rat model of severe chronic liver injury, suggesting that the therapeutic efficacy of MSCs needed to be improved.

The mode of therapeutic action of MSCs in liver disease was postulated to include differentiation into hepatocytes or fusion with endogenous hepatocytes. ${ }^{10}$ However, considering their low frequency of transdifferentiation or fusion, as well as the low numbers of transplanted MSCs, it is unlikely that MSC-derived hepatocytes could provide sufficient replacement of lost liver function. Moreover, MSCs do not only differentiate into hepatocytes or endothelial cells; a large fraction differentiates into fibrogenic myofibroblasts, leading to adverse effects. ${ }^{11-13}$

In addition to their differentiation potential, MSCs have the ability to supply beneficial cytokines. MSC-derived interleukin-10 and tumor necrosis factor- $\alpha$ inhibit the proliferation of Ito cells, and MSC-derived HGF was shown to induce the apoptosis of Ito cells in a co-culture system. ${ }^{14}$ Cytokines are produced in ample quantities by MSCs, thus supporting the view that cytokine production may be the major mode of therapeutic action of MSCs in liver fibrosis. This insight led us to postulate that augmentation of the production of therapeutic cytokines by MSCs would enhance their therapeutic efficacy in liver fibrosis.

HGF is a well-known anti-fibrotic cytokine, ${ }^{15}$ and delivery of the HGF gene or protein attenuates liver fibrosis in numerous in vivo models. ${ }^{16-19}$ The anti-fibrotic effect of HGF is thought to be achieved through attenuation of fibrogenic cytokine expression (transforming growth factor beta 1 (TGF- $\beta 1$ ) and platelet-derived growth factor-bb (PDGF-bb)), and through inhibition of the proliferation and activation of Ito cells, the major ECM producer in the liver. ${ }^{20}$ In addition, HGF inhibits the cell death of normal hepatocytes. ${ }^{21}$ The effectiveness and broad mode of action of this cytokine prompted us to choose HGF for testing the concept of enhancing cytokine production by MSCs used for cell therapy of liver fibrosis. In this report, we investigated the therapeutic efficacy of human MSCs ectopically overexpressing HGF (MSC/HGF) in a rat model of liver fibrosis induced by dimethylnitrosamine (DMN) injection.

\section{MATERIALS AND METHODS}

\section{Cell culture and gene transduction}

Human MSCs were isolated from bone marrow aspirate and expanded in culture as described previously. ${ }^{22}$ The adenoviral vector encoding human HGF (Ad-HGF) was kindly provided by $\mathrm{Dr}$ SJ Kim (ViroMed, Seoul, Korea). For adenoviral transduction, MSCs were exposed to fresh medium containing Ad-HGF (multiplicity of infection $=200$ ) for $1 \mathrm{~h}$. The medium was then removed, and the cells were washed once with DMEM and re-cultured in normal medium for $24 \mathrm{~h}$, after which cell transplantation was performed. Aliquots of transduced MSCs were maintained in culture for the determination of HGF production by transduced MSCs. The medium was sampled at 2, 4, 6,8 and 10 days after transduction and analyzed for HGF using a human HGF-specific ELISA kit (Institute of Immunology, Tokyo, Japan).

\section{Liver fibrosis animal model and cell transplantation}

Five-week-old, specific-pathogen-free, male Sprague-Dawley (SD) rats were obtained from Central Laboratory Animal Inc. (Seoul, Korea) and maintained in an air-conditioned animal house $\left(22^{\circ} \mathrm{C}, 55 \%\right.$ humidity, and $12: 12 \mathrm{~h}$ daylight/darkness cycles) in accordance with institutional guidelines. To induce liver fibrosis, rats $(n=65)$ were conditioned by intraperitoneal injection with $1 \%$ (V/V) DMN (Sigma-Aldrich, St. Louis, MO, USA) diluted in saline at a dose of $1 \mathrm{ml}$ per $\mathrm{kg}$ of body weight, on 3 consecutive days each week for 4 consecutive weeks; control rats $(n=10)$ were mock-conditioned by injection with saline. Liver biopsies were performed at 4 days after the last DMN administration for collagen determination. Collagen was determined by Sirius Red assay using SIRCOL Collagen Assay DYE reagent $(0.1 \%$ Sirius Red dye solution; Biocolor Ltd., Newtownabbey, UK) according to the manufacturer's guidelines. Briefly, extracts of biopsy samples were prepared by sonication in $0.5 \mathrm{M}$ acetic acid, and the extracts were incubated with Sirius Red for $30 \mathrm{~min}$. The absorbance of Sirius Red dye bound to collagen was measured at $550 \mathrm{~nm}$ to provide a quantitative indicator of the amount of collagen in the liver. Rats with a collagen level within one standard deviation from the average value were randomly divided into three groups and subjected to cell transplantation. DMN-conditioned rats were injected with saline or with MSCs or MSCs/HGF $\left(1 \times 10^{7}\right.$ cells per rat). Salineconditioned rats were injected with saline. The final groups were as follows (conditioning + therapeutic treatment): saline + saline, $n=7$; DMN + saline, $n=10$; DMN + MSCs, $n=10$; DMN + MSC/HGF, $n=11$.

\section{Analysis of liver tissue specimens and blood samples}

To directly compare liver biopsy samples with liver tissue obtained at killing, rats were numbered before biopsy and tracked individually until killing. Rats were killed 12 days after transplantation. For serum biochemical analysis, blood samples were collected from the portal vein and heart. Immediately after blood collection, alkaline phosphatase (ALP), alanine transaminase, aspartate transaminase, total bilirubin, direct bilirubin, albumin and $\gamma$-glutamyl transpeptidase concentrations were measured using a Toshiba 200FR automatic chemistry analyzer (Toshiba, Tokyo, Japan).

Liver tissue was excised from a distal site of the left lobe for Sirius red, reverse transcription PCR (RT-PCR) and western blot analysis. The remaining tissue was fixed and processed for histological and immunohistochemical analysis.

\section{Histological and immunohistochemical analysis}

Liver tissues were removed, post-fixed in 4\% PFA overnight, and then embedded in paraffin. For determination of liver fibrosis, 5 - $\mu \mathrm{m}$-thick paraffin sections were deparaffinized and stained with Picro-Sirius red. The ratio of the Sirius red-positive area to the total area was examined using the ImageJ $1.35 \mathrm{~s}$ software (National Institutes of Health, Bethesda, MD).

For detection of transplanted cells and HGF expression, deparaffinized liver sections were boiled in citrate buffer $(\mathrm{pH}$ 6.0) for $10 \mathrm{~min}$. After blocking in 5\% normal serum, the sections were incubated with anti-human HGF antibody (R\&D Systems, Minneapolis, MN, USA) and human mitochondria-specific antibody (hMt; Chemicon, Temecula, CA, USA) at $4{ }^{\circ} \mathrm{C}$ overnight. Sections were then incubated with AlexaFluor 488- or 594-conjugated anti-IgG secondary antibodies (Life Technologies, Grand Island, NY, USA) and counterstained with bis-benzamide (Molecular Probes, Eugene, OR, USA) to visualize 
nuclei. To detect Ito cells, sections were immunostained with antidesmin antibody (DAKO, Tokyo, Japan) or anti- $\alpha$-smooth muscle actin antibody ( $\alpha$-SMA; DAKO) and visualized using an ABC kit (Vector Laboratories, Burlingame, CA, USA) according to the manufacturer's instructions. Immunostained images were acquired using a Zeiss Axiophot microscope (Carl Zeiss, Jena, Germany).

\section{Hydroxyproline content assay}

The hydroxyproline content of the liver tissue was determined as described previously. ${ }^{23}$ Briefly, the samples were homogenized with a Polytron homogenizer (Kinematica Co., Luzern, Switzerland), followed by hydrolysis in $6 \mathrm{~N} \mathrm{HCl}$ at $120^{\circ} \mathrm{C}$ for $3 \mathrm{~h}$, and then dried in a rotary evaporator. Hydrolyzed samples were resuspended in buffer $\left(3.325 \%(\mathrm{~W} / \mathrm{V})\right.$ citric acid $1 \mathrm{H}_{2} \mathrm{O}, 0.08 \%(\mathrm{~V} / \mathrm{V})$ glacial acetic acid, $8 \%(\mathrm{~W} / \mathrm{V})$ sodium acetate $3 \mathrm{H}_{2} \mathrm{O}$, and $2.275 \%(\mathrm{~W} / \mathrm{V}) \mathrm{NaOH}$, $20 \%$ (V/V) 1-propanol) and then incubated with chloramine $\mathrm{T}$ solution $(50 \mu \mathrm{M})$ for $20 \mathrm{~min}$. The samples were then subjected to reaction with aldehyde-perchloric acid reagent (15\% (W/V) p-dimethylaminobenzaldehyde, 60\% (V/V) n-propanol, and $26 \%$ $(\mathrm{V} / \mathrm{V})$ perchloric acid) at $60^{\circ} \mathrm{C}$ for $15 \mathrm{~min}$. The absorbance of each sample was read at $550 \mathrm{~nm}$.

\section{Western blotting}

Liver tissues were lysed in RIPA buffer $(20 \mathrm{~mm}$ Tris, pH 7.4; $150 \mathrm{~mm} \mathrm{NaCl} ; 1 \%$ Triton $\mathrm{X}-100$; and $0.1 \%$ SDS) containing protease inhibitor $\left(10 \mu \mathrm{g} \mathrm{ml}^{-1}\right.$ leupeptin, $10 \mu \mathrm{g} \mathrm{ml}^{-1}$ aprotinin and $0.2 \mathrm{~mm}$ EDTA). Each liver lysate ( $50 \mu \mathrm{g}$ per sample) was resolved by 8 or $10 \%$ SDS-PAGE and the proteins were transferred onto a nitrocellulose membrane. Immunoblotting was performed with antibodies against $\alpha$-SMA (DAKO), desmin (DAKO), matrix metalloprotease-9 (MMP-9) (Biodesign, Kennebunk, ME, USA), or urokinase-type plasminogen activator (uPA) (Santa Cruz, Delaware Avenue, CA, USA); proteins were detected by enhanced chemical luminescence after incubation with appropriate horseradish peroxidase-conjugated secondary antibodies (Amersham, Arlington Height, IL, USA). The relative signal intensity of each lane was calculated using a GS-800 Calibrated Densitometer (Bio-Rad Laboratories, Hercules, CA, USA).

\section{Reverse transcription-PCR}

Total RNA from each sample was extracted using the Trizol Reagent (Life Technologies), and $3 \mu \mathrm{g}$ of RNA was used as the template for cDNA synthesis using AMV reverse transcriptase (TaKaRa Bio Inc., Shiga, Japan), according to the manufacturer's instructions. PCR amplification was subsequently performed using specific primers (summarized in Supplementary Table 1). PCR products were separated by $1.5 \%$ agarose gel electrophoresis, and the relative signal intensity of each lane was calculated using a GS-800 Calibrated Densitometer (Bio-Rad, Hercules, CA, USA). All PCRs were normalized to expression levels of GAPDH used as an internal standard.
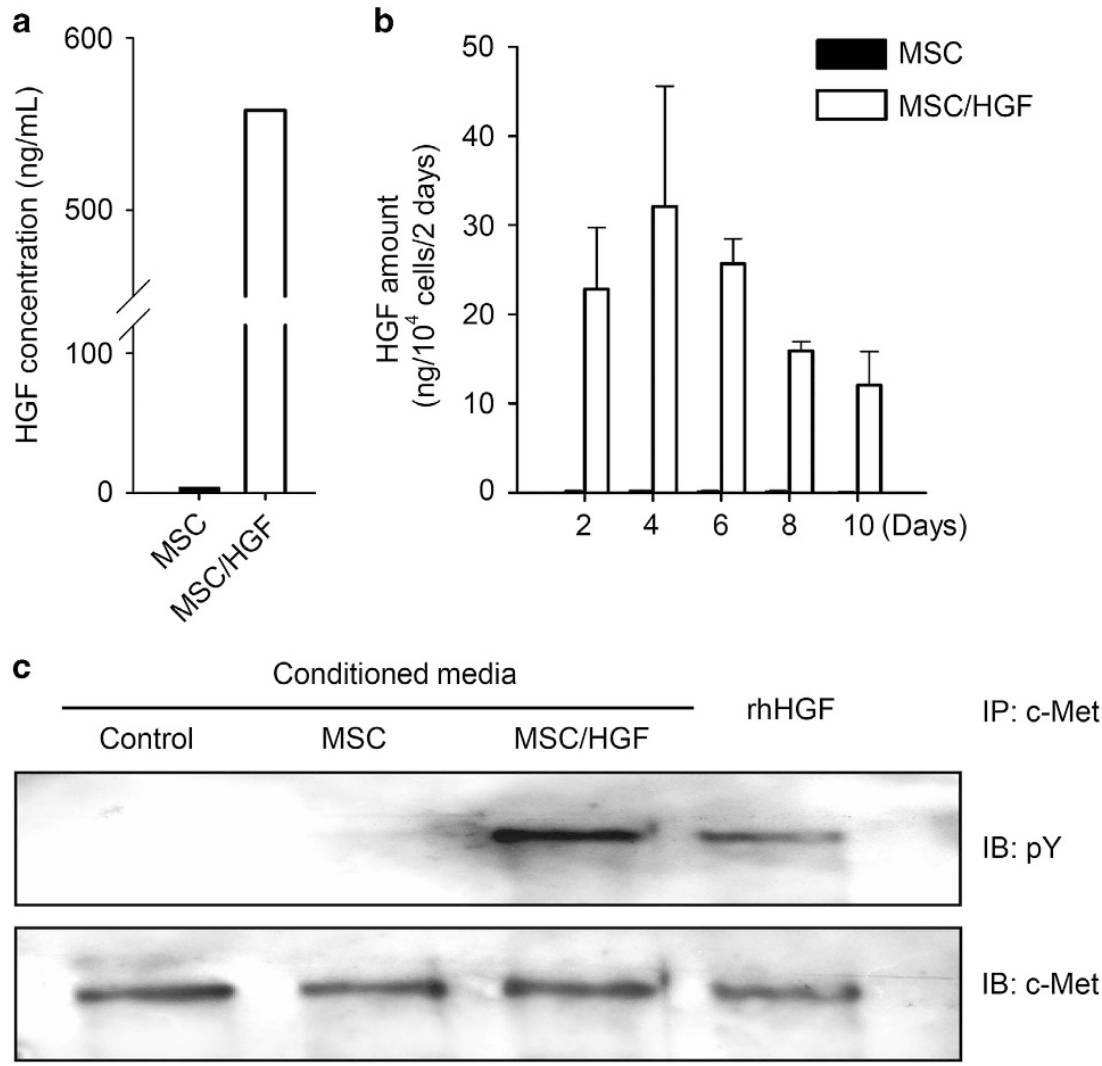

Figure 1 Analysis of HGF secreted by MSCs/HGF. (a) The amount of human HGF after 4 days of culture. (b) Secretion of HGF by MSCs or MSCs/HGF at 2, 4, 6, 8 and 10 days after Ad-HGF transduction. (c) Immunoblot showing c-Met phosphorylation in MDCK2 cells treated with conditioned media obtained from indicated cells. Human recombinant HGF protein (rhHGF, 100 unit $\mathrm{ml}^{-1}$ ) was used as a positive control. IB, immunoblotting; IP, immunoprecipitation; PY, phospho-tyrosine. 


\section{Statistical analysis}

Statistical analysis was performed using the SPSS 12.0.1. software (SPSS Inc., Chicago, IL, USA). All results are expressed as the mean \pm s.d. Comparisons between groups were performed using PROC ANOVA for one-way analysis and Scheffe's multiple-comparison procedure. $p<0.05$ was considered to be significant.

\section{RESULTS}

Quantification and functional assay of secreted HGF in vitro The amount of HGF produced by MSCs transduced with Ad-HGF was assessed by ELISA and was $\sim 160$ times higher than the amount endogenously secreted by MSCs (557.86 vs $3.49 \mathrm{ng} \mathrm{ml}^{-1}$, from $5 \times 10^{5}$ cells cultured for 4 days, Figure 1a). HGF production peaked at 2-4 days after transduction and was maintained for at least 10 days (Figure 1b). The biological activity of HGF produced by MSCs/HGF was confirmed by phosphorylation of c-Met, the receptor for HGF (Figure 1c), and by scattering of $\mathrm{MDCK}_{2}$ cells (data not shown), a characteristic function of HGF in these cells. ${ }^{24}$

\section{Enhanced resolution of liver fibrosis by transplantation of MSCs/HGF}

We adopted the DMN-induced rat liver fibrosis model in this study rather than the $\mathrm{CCl}_{4}$-induced model because $\mathrm{CCl}_{4}$-induced liver fibrosis resolves spontaneously. ${ }^{25}$ To address the therapeutic effect rather than the preventive effect, we injected saline, MSCs, or MSCs/HGF into the spleens of the rats once on the ninth day after the final DMN injection (4 days after biopsy; Figure $2 \mathrm{a}$ ). The average collagen content (Figure 2c) and average body weight (Figure 2e) of all DMN-treated animals were similar at the time of cell transplantation, indicating that the extent of fibrosis was not different before transplantation. The rats were killed at 12 days after cell transplantation, and fibrosis

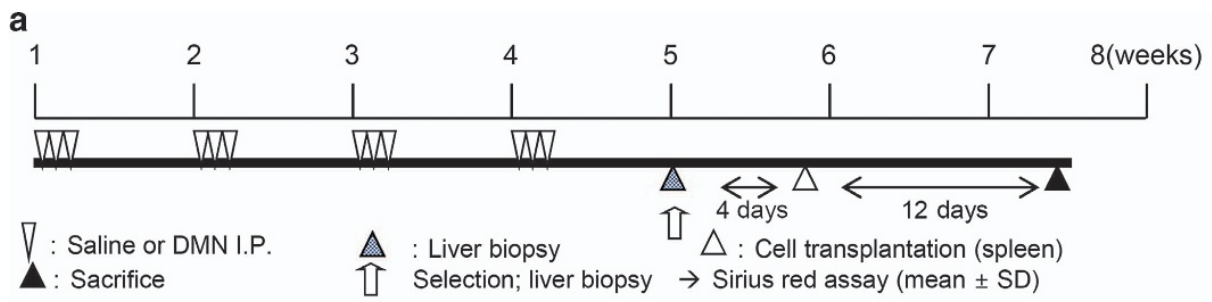

b
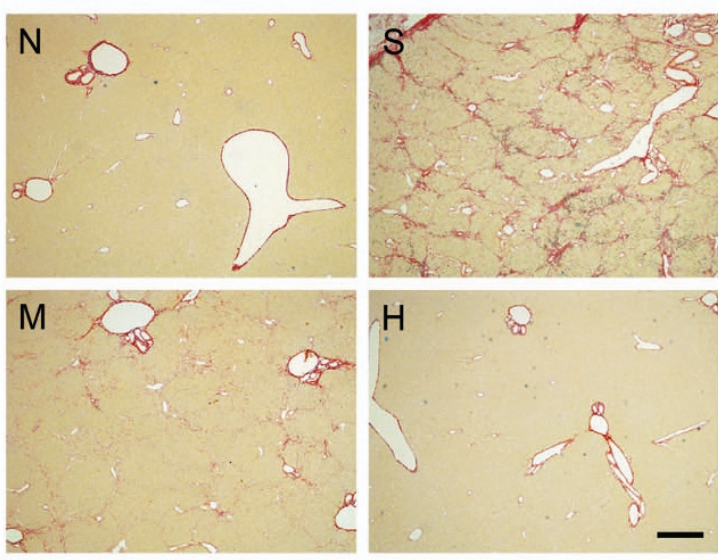

d

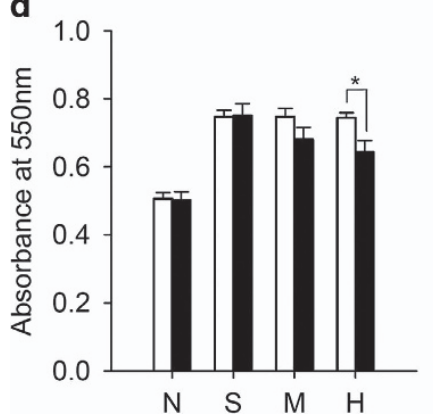

$\mathrm{H}=\infty$

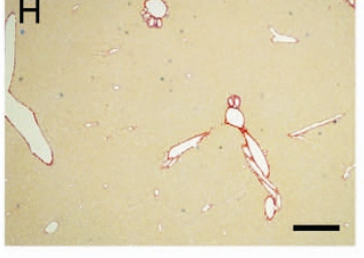

c

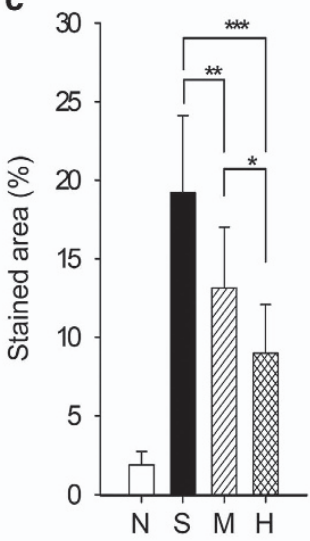

e

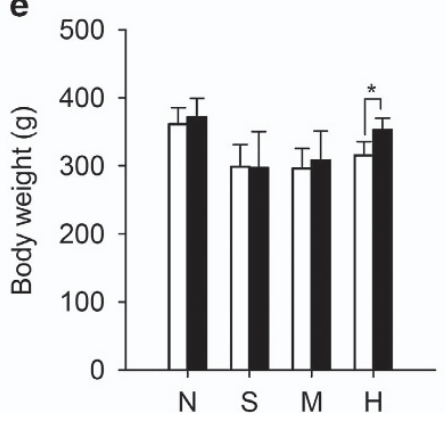

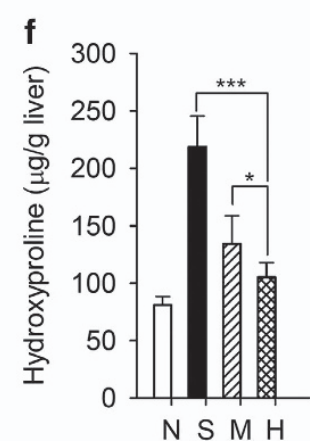

Figure 2 Assessment of liver fibrosis in MSC/HGF-transplanted rats and controls. (a) Diagram of the treatment protocol. (b) Extracellular deposition of collagen fibers stained with Sirius Red. Scale bar $=0.5 \mathrm{~mm}$. (c) Quantification of collagen by image analysis. (d, e) Collagen content and body weight quantified before (white bar) and 12 days after transplantation (black bar). (f) Hydroxyproline content. Normal rats $(N)$, control fibrotic animals $(S)$, fibrotic animals transplanted with MSCs $(M)$ or MSCs/HGF $(H)$. Data represent mean \pm s.d. for each group ( ${ }^{*} P<0.05,{ }^{* *} P<0.01,{ }^{* *} P<0.005$ ). 
was evaluated. Liver fibrosis was evident based on the formation of fibrotic septa joining the central area (Figure 2b, right upper panel). Quantitative measurement of collagen in liver tissue by Sirius red assay also revealed increased collagen content (Figure 2d, normal, $0.506 \pm 0.019$ vs saline/DMN, $0.748 \pm 0.018, \quad P<0.001)$, which was consistent with the histological changes. The increased collagen content of the rat livers was sustained during the entire experimental period after completion of DMN conditioning, indicating that the fibrosis in this experimental system was stable (Figure 2d, liver biopsy, $0.748 \pm 0.018$ vs killing, $0.751 \pm 0.035$ in the saline/DMN group).

The collagen-stained area in liver sections was significantly decreased by MSC transplantation and was further reduced by MSC/HGF transplantation (Figure 2c, saline, $19.2 \pm 4.90 \%$ vs MSCs, $13.14 \pm 3.88 \%$ vs MSCs/HGF, $9.0 \pm 3.09 \%$; saline vs MSCs $P<0.05$; saline vs MSCs/HGF $P<0.001 ;$ MSCs vs MSCs/HGF $P<0.05)$. Tissue hydroxyproline levels, an another indicator of tissue collagen content, ${ }^{26}$ showed a similar pattern (Figure 2f, saline, $218.7 \pm 26.8 \mu \mathrm{gg}^{-1}$, MSCs, $134.2 \pm 24.3 \mu \mathrm{gg}^{-1}$, MSCs/HGF, $105.2 \pm 12.6 \mu \mathrm{gg}^{-1}$; saline vs MSCs $P<0.001$, MSCs vs MSC/HGF $P<0.05)$. Surprisingly, when we compared the tissues at the time of biopsy with tissues at the time of killing, the amount of collagen was decreased in the MSC group and in the MSC/HGF group, whereas the amount of collagen in the control and saline/DMN groups did not change (Figures $2 \mathrm{c}$ and $\mathrm{d}$ ), suggesting that resolution of fibrosis was occurring. We also evaluated changes in body weight as a parameter reflecting the general condition of the rats. Body weight was significantly increased only in the MSC/HGF group $(P<0.05$, Figure 2e). These data clearly demonstrate that MSCs/ HGF were significantly more effective than MSCs alone as a therapeutic for rat liver fibrosis.
Functional recovery from DMN-induced liver damage by transplantation of MSCs/HGF

We next evaluated the effects of cell transplantation on the extent of liver injury and liver function. Serum transaminase levels, which increase when the liver is damaged, were significantly attenuated in the MSC/HGF group (Figures 3a and b), as were ALP levels, which increase when biliary epithelial cells are damaged (Figure 3c), compared with the MSC group and saline control group, demonstrating that MSCs/HGF were more effective in preventing liver damage associated with fibrosis. Similarly, the serum ammonia level, which is an indicator of urea cycle function in hepatocytes, was normalized in the MSC/HGF group to a greater extent than in the MSC group (Figure 3d). In addition, the serum albumin level, which is an indicator of liver function, was slightly increased in the MSC/HGF group but not in the MSC or saline control group $(P<0.05$; Figure $3 \mathrm{e})$. Taken together, our data demonstrate that MSCs/HGF are more beneficial than MSCs alone with respect to the attenuation of liver damage and recovery of liver function.

\section{Detection of transplanted cells and determination of human HGF levels in vivo}

Our model system was based on the xenotransplantation of human MSCs into rats in the absence of immunosuppression. Thus, we verified the presence of transplanted human cells and the production of human HGF at the time of killing. Because we injected the cells into the spleen, we assumed that the transplanted cells would act directly in the liver after migration or would act indirectly by secreting HGF into the bloodstream, which would eventually reach the liver via the portal vein. The human HGF level in the portal vein was measured by human HGF-specific ELISA. As shown in Figure $4 \mathrm{a}$, human HGF was detected in eight of nine rats of a
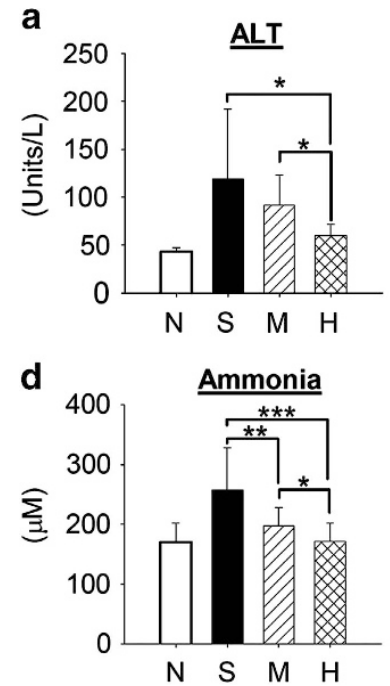

b

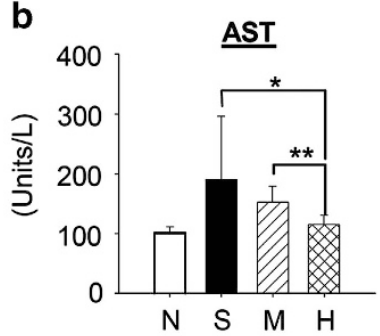

e

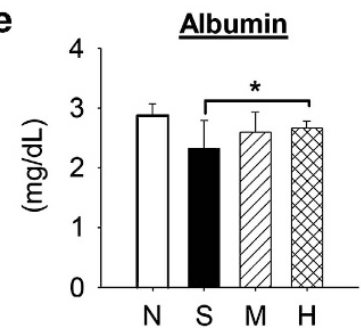

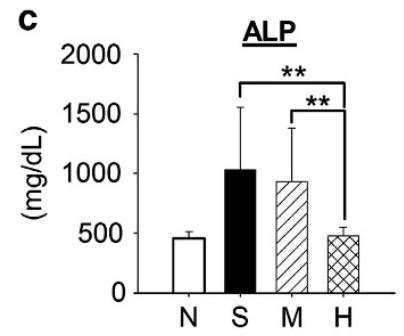
Figure 3 Examination of serum parameters. (a, b) Transaminases (ALT and AST), (c) alkaline phosphatase (ALP), (d) ammonia and
(e) albumin. Normal rats, $N$; fibrotic control animals, $S$; fibrotic animals transplanted with MSCs, M, or MSCS/HGF, H. Data represent the mean \pm s.d. for each group $\left({ }^{*} P<0.05,{ }^{* * P}<0.01,{ }^{* * * P}<0.005\right)$. 

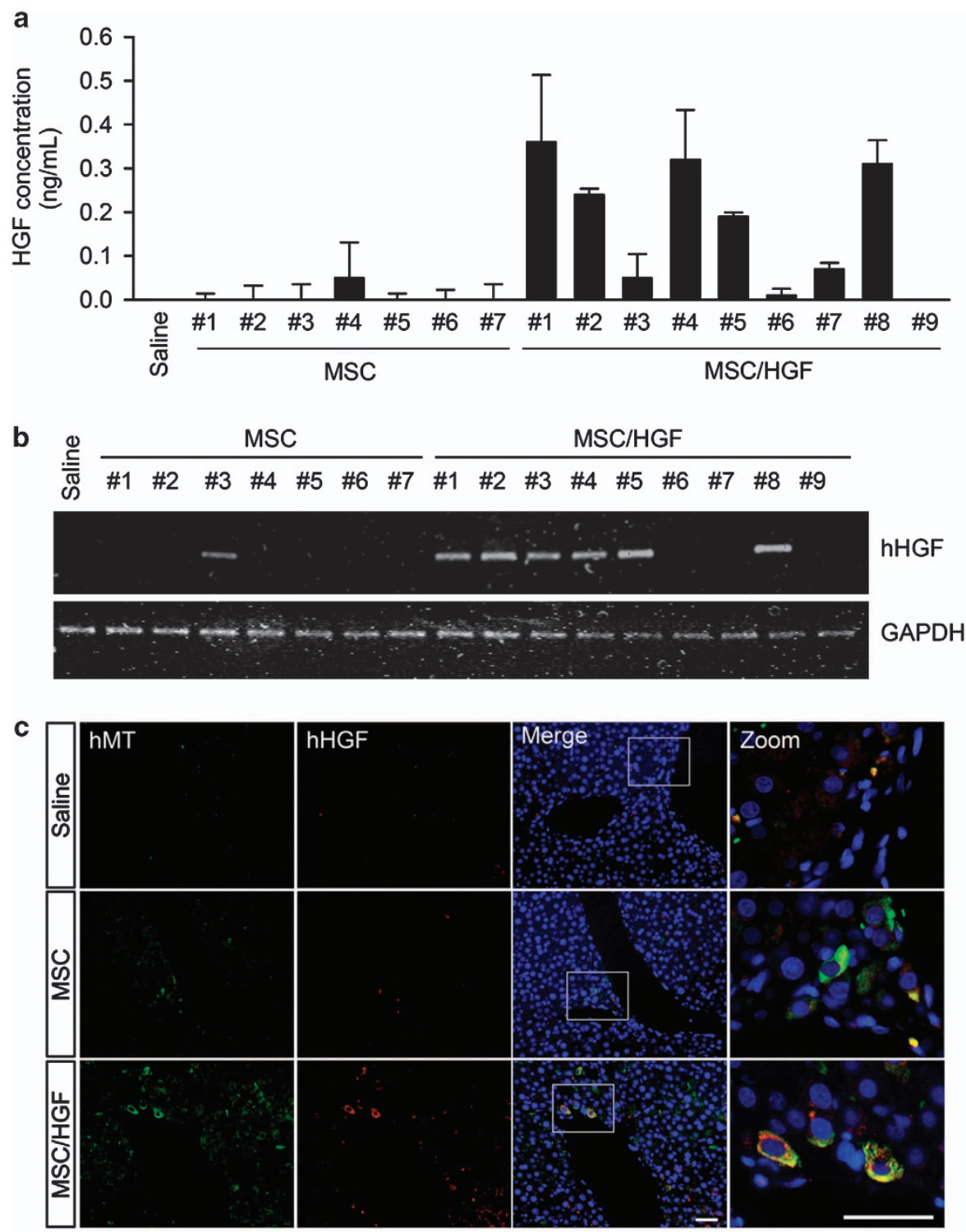

Figure 4 Analysis of human HGF expression in rats transplanted with MSCs/HGF. (a) Human HGF proteins. Values represent the mean \pm s.d. of triplicate assays of individual rats. (b) Human HGF mRNA. (c) Immunostaining for human mitochondria-specific antigen (hMT) and human HGF (hHGF). Blue: bis-benzamide, green: hMT, red: hHGF, Scale bar, $50 \mu \mathrm{m}$. Samples are fibrotic livers from control animals or from animals transplanted with MSCs or MSCs/HGF except in (a), where analysis was performed on serum collected from the portal vein 12 days after transplantation.

the MSC/HGF group, and in only one of seven rats in the MSC group. In addition, the average level of HGF in the portal vein was significantly higher in the MSC/HGF group than in the MSC group (MSC, $0.01 \pm 0.018 \mathrm{ng} \mathrm{ml}^{-1}$ vs MSC/ HGF, $0.19 \pm 0.154 \mathrm{ng} \mathrm{ml}^{-1}$ ), indicating that a considerable amount of human HGF produced by cells transplanted into the spleen was delivered to the liver during the experimental period.

We next examined the presence of transplanted cells in the liver and assessed their function in terms of HGF production. Expression of human HGF mRNA was detected by semiquantitative RT-PCR in the liver tissue of six of nine rats transplanted with MSCs/HGF (Figure 4b). In contrast, human
HGF mRNA was detected in only one of eight rats transplanted with MSCs. This difference can likely be attributed to the difference in HGF expression level between MSCs and MSCs/HGF. Therefore, the presence of the transplanted cells in the liver tissue was directly evaluated by immunohistochemistry using antibodies against human mitochondria-specific antigen or human HGF (Figure 4c). Although the background fluorescence signal was somewhat high, we could clearly recognize double-positive cells (human mitochondrial antigen and human HGF) in tissue sections from both MSC and MSC/ HGF groups. The intensity of staining for human HGF was clearly higher in the MSC/HGF group than in the MSC group. It was remarkable that the xenotransplanted human MSCs 
survived for 12 days and produced human HGF without immunosuppression.

Decreased expression of profibrogenic cytokines and inactivation of Ito cells by MSCs/HGF

We next tried to elucidate the mechanistic basis for the enhanced therapeutic effect of transplanted MSCs/HGF. Because HGF was reported to downregulate TGF- $\beta 1$ and PDGF-bb, we assessed the expression of TGF- $\beta 1$ and PDGFbb mRNA in the liver (Figure 5a). Although mRNA levels varied among animals, expression of TGF- $\beta 1$ and PDGF-bb was significantly increased in fibrotic livers, and this effect was dramatically ameliorated by MSCs/HGF but not by MSCs (Figures $5 \mathrm{~b}$ and $\mathrm{c}$ ).

HGF has been reported to directly affect the survival and activation of rat Ito cells in vivo. ${ }^{20}$ Therefore, we assessed the effect of MSCs/HGF and MSCs on Ito cells by measuring $\alpha$ SMA, which is a marker of activated Ito cells, and desmin, which is a marker of Ito cells irrespective of their activation state. $^{27}$ Immunohistochemistry revealed that $\alpha$-SMA and desmin expression decreased more in the MSC/HGF group than in the MSC group (Figure 6a). Western blotting of pooled liver lysates from each group further confirmed this observation (Figure 6b). In addition, the calculated expression of $\alpha$-SMA and desmin protein for each experimental animal was similar to that in pooled samples (Figure $6 \mathrm{c}$ ). Based on these results, we conclude that MSCs/HGF decreased both the number and the activation of Ito cells to a greater extent than MSCs, most probably as a result of the known inhibitory activity of HGF on the survival and activation of Ito cells.

Effect on ECM remodeling molecules in fibrosis resolution The decreased collagen content in the liver after MSC/HGF transplantation suggested that there was an active remodeling of ECM components in the liver following MSC/HGF transplantation. Because the amount of ECM depends on the balance between production and degradation, we investigated the expression of Type 1 collagen, MMPs and tissue inhibitor of metalloproteases (TIMPs) in our system. The mRNA level of Type 1 collagen, a representative ECM molecule, was significantly decreased in the MSC/HGF group (Figure 7a).

MMPs and TIMPs are key regulators of ECM remodeling, ${ }^{28}$ and many ECM glycoproteins, such as fibronectin and laminins, are degraded by MMP-2 and MMP-9, ${ }^{29}$ whereas MMP-2 activity is regulated by TIMP-2 and MMP-14 via a

a $\mathrm{N}$

S

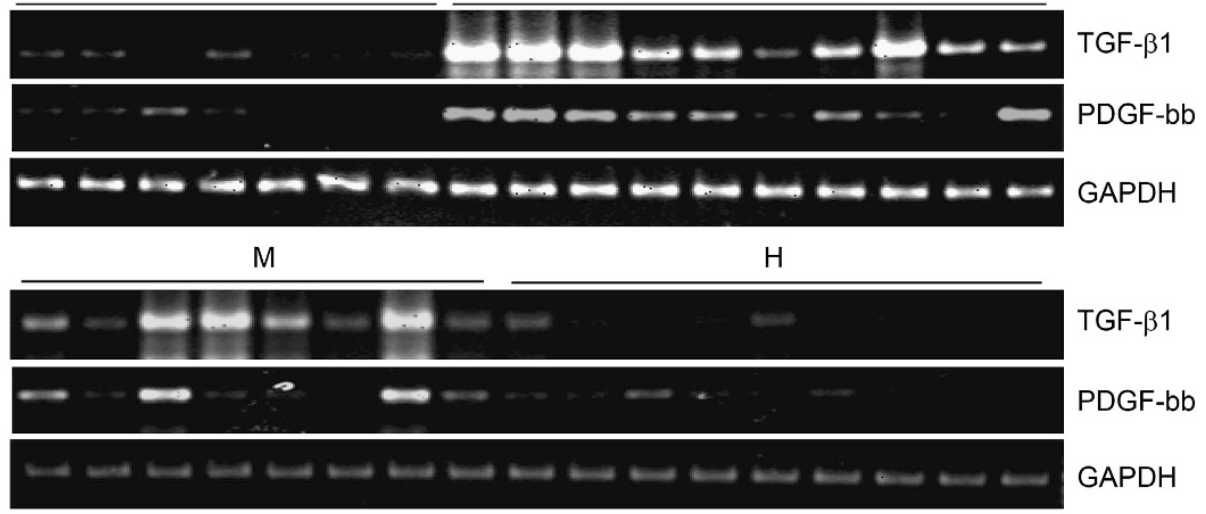

b

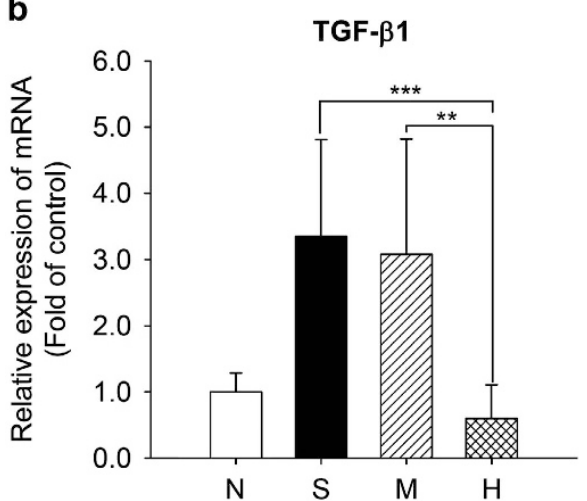

C

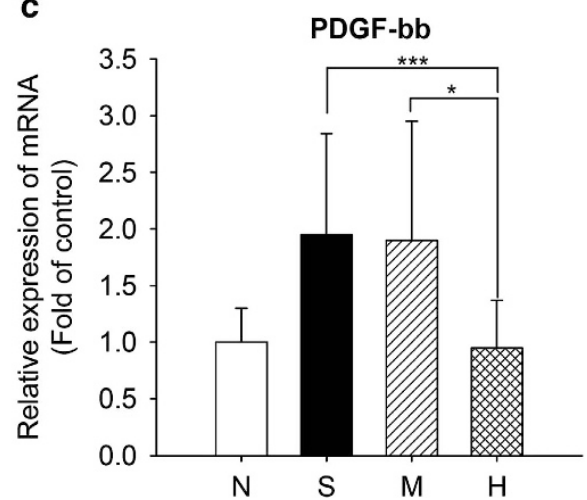

Figure 5 Analysis of profibrogenic cytokine expression. (a) Detection of TGF- $\beta 1$ and PDGF-bb mRNA in individual rats by RT-PCR. (b, c) Mean relative expression levels of TGF- $\beta 1$ and PDGF-bb in liver tissues from normal rats, $N$; control fibrotic animals, $S$; or fibrotic animals transplanted with MSCs, $M$, or MSC/HGF, $H$. Data are normalized to GAPDH expression levels and represent the mean $\pm \mathrm{s}$.d. for each group $\left({ }^{*} P<0.05,{ }^{* *} P<0.01,{ }^{* * *} P<0.005\right)$. 
a

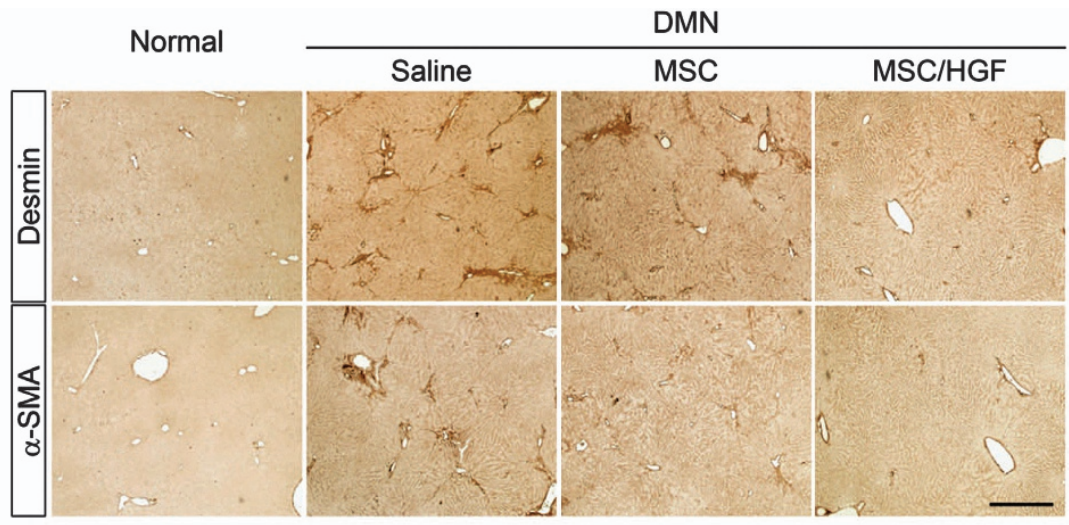

b

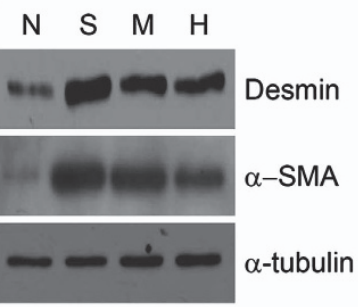

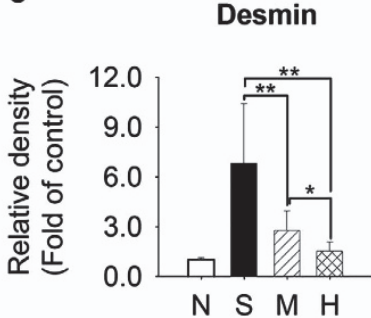

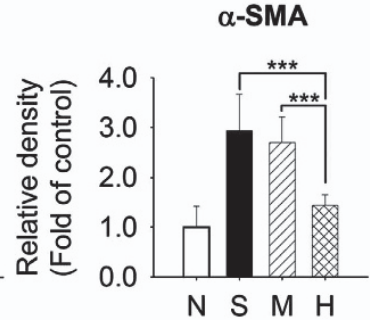

Figure 6 Effect of MSC/HGF transplantation on Ito cell activation. (a) $\alpha$-SMA and desmin were detected by immunohistochemistry in liver sections. Scale bar, $500 \mu \mathrm{m}$. (b) Liver extracts from each group were subjected to western blot analysis using antibodies against $\alpha$-SMA and desmin. (c) The relative amount of $\alpha$-SMA and desmin proteins was calculated by densitometry. Normal rats, $N$; control fibrotic animals, $S$; and fibrotic animals transplanted with MSCs, M, or MSCs/HGF, $H$. Data represent the mean \pm s.d. for each group. $\alpha$-SMA; $\alpha$-smooth muscle actin ( $\left.{ }^{*} P<0.05,{ }^{* *} P<0.01,{ }^{* *} P<0.005\right)$.

tri-molecular interaction. ${ }^{28}$ In the present study, the expression of TIMP-2 and MMP-2 mRNA did not change (Figures 7c and d), whereas the reduction in MMP-14 mRNA caused by DMN administration was reversed only in the MSC/HGF group (Figure $7 \mathrm{~g}$ ). In contrast, MMP-13, which degrades interstitial collagen and is expressed in the recovery phase of liver fibrosis, ${ }^{30}$ was upregulated in both MSC and MSC/HGF groups (Figure 7f). TIMP-1 was downregulated only in the MSC/HGF group (Figure 7b). uPA generates plasmin, which then activates MMP-9. ${ }^{28}$ Protein and mRNA expression of MMP-9 (Figures 7e, h and i) and protein expression of uPA (Figures $7 \mathrm{~h}$ and $\mathrm{j}$ ) were increased in both MSC and MSC/HGF groups.

\section{DISCUSSION}

In this study, we found that MSCs that overexpress HGF have an enhanced therapeutic effect compared with non-manipulated MSCs in a rat liver fibrosis model. Recent studies have shown that the potential of MSCs to differentiate into liver parenchymal cells is not sufficient to fully resolve liver fibrosis. Furthermore, di Bonzo et al. ${ }^{13}$ suggested that MSCs are a twoedged sword in hepatic regenerative medicine, as they can differentiate into either hepatocytes or profibrogenic myofibroblasts. Similarly, Aswa et al. ${ }^{31}$ suggested inhibiting migration of bone marrow cells to the liver as a strategy for prevention of biliary fibrosis. Nonetheless, several animal and clinical studies have reported beneficial effects of MSCs in the treatment of liver fibrosis, suggesting modes of action distinct from differentiation into parenchymal cells, such as alteration of environmental conditions in the injured tissue through production of soluble factors. Given that MSCs have been demonstrated to produce a wide array of trophic and growth factors both in vivo and in vitro, a reasonable explanation for the functional benefit derived from MSCs may be that they produce organotrophic factors that protect cells from damage or activate endogenous restorative mechanisms within the injured tissue.

The clinical transplantation of organs and cells is limited practically by the availability of donors. Organs and cells to be transplanted must not only be available in sufficient quantity but must also be immunologically compatible with the recipient. Allotransplantation requires immunosuppression in addition to matching of the donor and the recipient by tissue typing. One noteworthy aspect of the present study was that although this study involved xenotransplantation, we did not use immunosuppression. MSCs are reported to escape immune recognition and to inhibit immune responses through inhibition of the activation and differentiation of immune cells such as antigen-presenting cells, $\mathrm{T}$ cells, $\mathrm{B}$ cells and natural killer cells. ${ }^{32-34}$ Moreover, HGF is known to induce immunological tolerance and has been reported to suppress T-cell proliferation and dendrite cell antigen presentation, reduces acute and chronic allograft rejection, ameliorates the progression of experimental autoimmune myocarditis, and attenuates allergic airway inflammation. ${ }^{35-38}$ In the current study, the two immune modulatory factors, MSCs and HGF, 

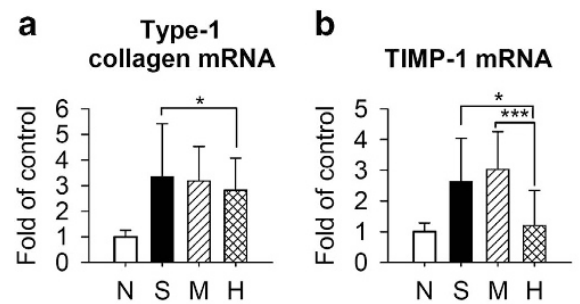

C

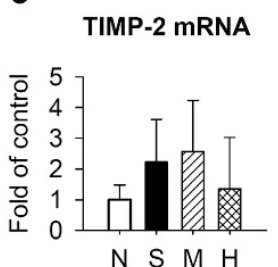

d

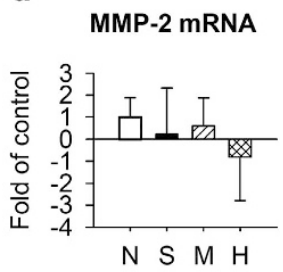

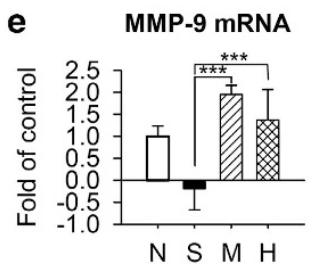
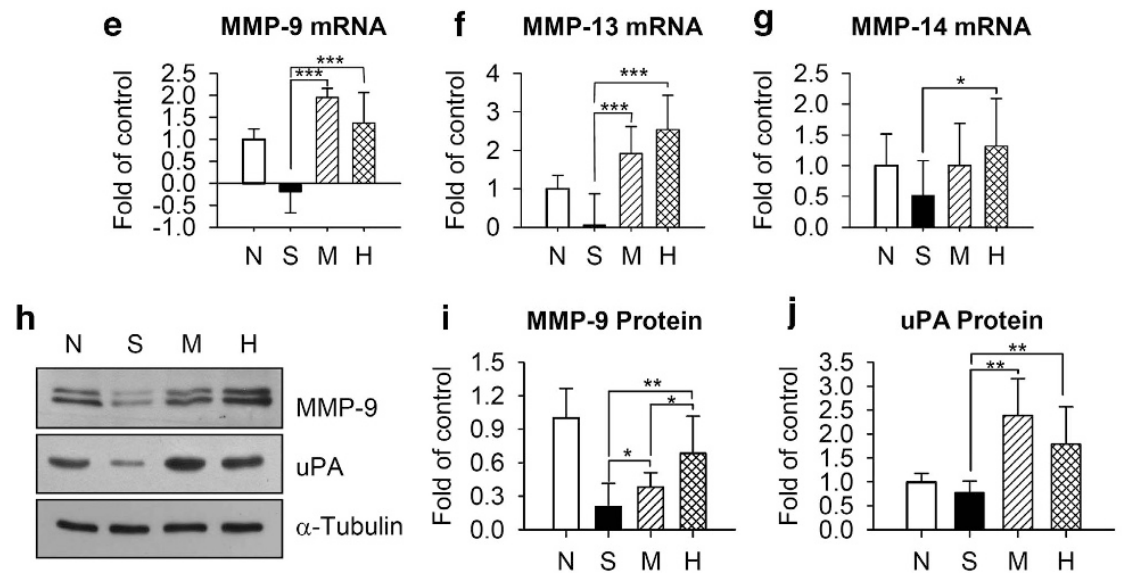

Figure 7 Analysis of the expression of ECM remodeling factors. (a) Type 1 collagen; (b, c) TIMP-1 and -2; and (d-g) MMP-2, -9, -13 and -14 mRNAs were quantified by semiquantitative RT-PCR. (h) MMP-9 and uPA protein levels were evaluated by western blotting and (i, j) quantified by densitometric analysis. Samples were obtained from normal rats, $N$; control fibrotic animals, $S$; fibrotic animals transplanted with MSCs, $M$, or MSCs/HGF, $H$. Data represent the mean \pm s.d. for each group. MMP, matrix metalloproteinase; TIMP, tissue inhibitor of matrix metalloproteinase, uPA, urokinase type plasminogen activator $\left({ }^{*} P<0.05,{ }^{*} P<0.01,{ }^{* *} P<0.005\right)$.

appear to have played important roles in maintaining a significant population of transplanted cells during the treatment period in the absence of additional immunosuppression. MSCs/HGF may therefore be suitable for treatment of liver fibrosis irrespective of the immunological phenotype of the patient.

Frequently, chronic diseases, including liver fibrosis, are not diagnosed until the condition has reached an advanced stage. Therefore, therapeutic drugs, rather than preventive drugs, are promising and practical from a clinical perspective. In this experiment, MSCs or MSCs/HGF as therapeutic candidates were transplanted after liver fibrosis had been induced by DMN administration. We adopted the DMN model because it has been found to be relatively stable ${ }^{39}$ but may progress after administration of DMN ceases. ${ }^{25}$ In the current study, we initiated cell therapy after liver fibrosis was established and stabilized, as indicated by the comparable collagen content in the liver at biopsy and at killing among control animals. Taken together, the results indicate that MSCs/HGF may be a favorable therapeutic modality for treatment of liver fibrosis.

In this study, HGF was delivered by MSCs, but the results were comparable to data from studies in which HGF protein was injected directly. ${ }^{21}$ Our data show that MSC/HGF treatment affected most relevant parameters in producing an anti-fibrotic effect. We found several strong correlations among parameters, including, for example, HGF level vs MMP-13 level $(R=0.77)$. These correlations provide clues regarding possible causal relationships between parameters that need further investigation.

In summary, the present study shows that cell therapy with MSCs/HGF significantly promotes liver function and attenuates liver fibrosis. These effects are superior to treatment with MSCs alone. The enhanced therapeutic effects of MSCs/HGF were accompanied by decreased levels of the fibrogenic cytokines TGF- $\beta 1$ and PDGD-bb, which may in turn be responsible for the observed reduction in the number and activation of Ito cells. Transplantation with MSCs/HGF also resulted in a decrease in MMP expression and an increase in TIMP expression. Cell therapy with MSCs/HGF may be a new and effective tool for the treatment of fibrotic liver disease.

\section{CONFLICT OF INTEREST}

The authors declare no conflict of interest.

\section{ACKNOWLEDGEMENTS}

This study was partly supported by a grant of the Health \& Medical Technology R\&D program, Ministry of Health \& Welfare, Republic of Korea (A050343 to J-HL); by the National Research Foundation of Korea (NRF) grant funded by the Korea government (MSIP) (No. 2011-0030043 to J-HL) and the Bio \& Medical Technology Development Program of the National Research Foundation (NRF) funded by the Ministry of Science, ICT \& Future Planning (NRF-2012M3A9C6049725 to S-SK \& NRF-2010-0020406 to HS-K). 
1 Bataller R, Brenner DA. Liver fibrosis. J Clin Invest 2005; 115: 209-218.

2 Sakaida I, Terai S, Yamamoto N, Aoyama K, Ishikawa T, Nishina H et al. Transplantation of bone marrow cells reduces $\mathrm{CCl}$-induced liver fibrosis in mice. Hepatology 2004; 40: 1304-1311.

3 Fang B, Shi M, Liao L, Yang S, Liu Y, Zhao RC. Systemic infusion of FLK1(+) mesenchymal stem cells ameliorate carbon tetrachlorideinduced liver fibrosis in mice. Transplantation 2004; 78: 83-88.

4 Oyagi S, Hirose M, Kojima M, Okuyama M, Kawase M, Nakamura T et al. Therapeutic effect of transplanting HGF-treated bone marrow mesenchymal cells into CCl4-injured rats. J Hepatol 2006; 44: 742-748.

5 Ong SY, Dai H, Leong KW. Hepatic differentiation potential of commercially available human mesenchymal stem cells. Tissue Eng 2006; 12: 3477-3485.

6 Lee KD, Kuo TK, Whang-Peng J, Chung YF, Lin CT, Chou SH et al. In vitro hepatic differentiation of human mesenchymal stem cells. Hepatology 2004; 40: 1275-1284.

7 Ong SY, Dai H, Leong KW. Inducing hepatic differentiation of human mesenchymal stem cells in pellet culture. Biomaterials 2006; 27: 4087-4097.

8 Zhan Y, Wang Y, Wei L, Chen H, Cong X, Fei R et al. Differentiation of hematopoietic stem cells into hepatocytes in liver fibrosis in rats. Transplant Proc 2006; 38: 3082-3085.

9 Carvalho AB, Quintanilha LF, Dias JV, Paredes BD, Mannheimer EG, Carvalho FG et al. Bone marrow multipotent mesenchymal stromal cells do not reduce fibrosis or improve function in a rat model of severe chronic liver injury. Stem Cells 2008; 26: 1307-1314.

10 Kallis YN, Alison MR, Forbes SJ. Bone marrow stem cells and liver disease. Gut 2007; 56: 716-724.

11 Russo FP, Alison MR, Bigger BW, Amofah E, Florou A, Amin F et al. The bone marrow functionally contributes to liver fibrosis. Gastroenterology 2006; 130: 1807-1821.

12 Forbes SJ, Russo FP, Rey V, Burra P, Rugge M, Wright NA et al. A significant proportion of myofibroblasts are of bone marrow origin in human liver fibrosis. Gastroenterology 2004; 126: 955-963.

13 di Bonzo LV, Ferrero I, Cravanzola C, Mareschi K, Rustichell D, Novo E et al. Human mesenchymal stem cells as a two-edged sword in hepatic regenerative medicine: engraftment and hepatocyte differentiation versus profibrogenic potential. Gut 2008; 57: 223-231.

14 Parekkadan B, van Poll D, Megeed Z, Kobayashi N, Tilles AW, Berthiaume $\mathrm{F}$ et al. Immunomodulation of activated hepatic stellate cells by mesenchymal stem cells. Biochem Biophys Res Commun 2007; 363: 247-252.

15 Mizuno S, Nakamura T. Hepatocyte growth factor: a regenerative drug for acute hepatitis and liver cirrhosis. Regen Med 2007; 2: 161-170.

16 Matsuno Y, Iwata H, Umeda Y, Takagi H, Mori Y, Kosugi A et al. Hepatocyte growth factor gene transfer into the liver via the portal vein using electroporation attenuates rat liver cirrhosis. Gene Ther 2003; 10 $1559-1566$.

17 Ueki T, Kaneda Y, Tsutsui H, Nakanishi K, Sawa Y, Morishita R et al. Hepatocyte growth factor gene therapy of liver cirrhosis in rats. Nat Med 1999; 5: 226-230.

18 Matsuda Y, Matsumoto K, Yamada A, Ichida T, Asakura H, Komoriya Y et al. Preventive and therapeutic effects in rats of hepatocyte growth factor infusion on liver fibrosis/cirrhosis. Hepatology 1997; 26: 81-89.

19 Yasuda H, Imai E, Shiota A, Fujise N, Morinaga T, Higashio K. Antifibrogenic effect of a deletion variant of hepatocyte growth factor on liver fibrosis in rats. Hepatology 1996; 24: 636-642.

20 Kim WH, Matsumoto K, Bessho K, Nakamura T. Growth inhibition and apoptosis in liver myofibroblasts promoted by hepatocyte growth factor leads to resolution from liver cirrhosis. Am J Pathol 2005; 166: 1017-1028.

21 Ishii T, Sato M, Sudo K, Suzuki M, Nakai H, Hishida T et al. Hepatocyte growth factor stimulates liver regeneration and elevates blood protein level in normal and partially hepatectomized rats. J Biochem (Tokyo) 1995; 117: 1105-1112.
22 Kim SS, Yoo SW, Park TS, Ahn SC, Jeong HS, Kim JW et al. Neura induction with neurogenin 1 increases the therapeutic effects of mesenchymal stem cells in the ischemic brain. Stem Cells 2008; 26: 2217-2228.

23 Edwards CA, O'Brien Jr WD. Modified assay for determination of hydroxyproline in a tissue hydrolyzate. Clin Chim Acta 1980; 104: 161-167.

24 Balkovetz DF. Hepatocyte growth factor and Madin-Darby canine kidney cells: in vitro models of epithelial cell movement and morphogenesis. Microsc Res Tech 1998; 43: 456-463.

25 Tsukamoto H, Matsuoka M, French SW. Experimental models of hepatic fibrosis: a review. Semin Liver Dis 1990; 10: 56-65.

26 Udenfriend S. Formation of hydroxyproline in collagen. Science 1966; 152: 1335-1340.

27 Friedman SL. Molecular regulation of hepatic fibrosis, an integrated cellular response to tissue injury. J Biol Chem 2000; 275: 2247-2250.

28 Arthur MJ. Fibrogenesis II. Metalloproteinases and their inhibitors in liver fibrosis. Am J Physiol Gastrointest Liver Physiol 2000; 279: G245-G249.

29 Grigorescu M. Noninvasive biochemical markers of liver fibrosis. J Gastrointest Liver Dis 2006; 15: 149-159.

30 Okazaki I, Watanabe T, Hozawa S, Niioka M, Arai M, Maruyama K. Reversibility of hepatic fibrosis: from the first report of collagenase in the liver to the possibility of gene therapy for recovery. Keio J Med 2001; 50: 58-65.

31 Asawa S, Saito T, Satoh A, Ohtake K, Tsuchiya T, Okada $\mathrm{H}$ et al. Participation of bone marrow cells in biliary fibrosis after bile duct ligation. J Gastroenterol Hepatol 2007; 22: 2001-2008.

32 Aggarwal S, Pittenger MF. Human mesenchymal stem cells modulate allogeneic immune cell responses. Blood 2005; 105: 1815-1822.

33 Inoue S, Popp FC, Koehl GE, Piso P, Schlitt HJ, Geissler EK et al. Immunomodulatory effects of mesenchymal stem cells in a rat organ transplant model. Transplantation 2006; 81: 1589-1595.

34 Sotiropoulou PA, Perez SA, Gritzapis AD, Baxevanis CN, Papamichail M. Interactions between human mesenchymal stem cells and natural killer cells. Stem Cells 2006; 24: 74-85.

35 Futamatsu H, Suzuki J, Mizuno S, Koga N, Adachi S, Kosuge H et al. Hepatocyte growth factor ameliorates the progression of experimental autoimmune myocarditis: a potential role for induction of $\mathrm{T}$ helper 2 cytokines. Circ Res 2005; 96: 823-830.

36 Okunishi K, Dohi M, Nakagome K, Tanaka R, Mizuno S, Matsumoto K et al. A novel role of hepatocyte growth factor as an immune regulator through suppressing dendritic cell function. J Immunol 2005; 175: 4745-4753.

37 Ito W, Kanehiro A, Matsumoto K, Hirano A, Ono K, Maruyama H et al. Hepatocyte growth factor attenuates airway hyperresponsiveness, inflammation, and remodeling. Am J Respir Cell Mol Biol 2005; 32: 268-280.

38 Yamaura K, Ito K, Tsukioka K, Wada Y, Makiuchi A, Sakaguchi M et al. Suppression of acute and chronic rejection by hepatocyte growth factor in a murine model of cardiac transplantation: induction of tolerance and prevention of cardiac allograft vasculopathy. Circulation 2004; 110 1650-1657.

39 Madden JW, Gertman PM, Peacock Jr EE. Dimethylnitrosamine-induced hepatic cirrhosis: a new canine model of an ancient human disease. Surgery 1970; 68: 260-267; discussion 267-8.

(c) (1) ()(2) This work is licensed under a Creative Commons Attribution-NonCommercial-ShareAlike 3.0 Unported License. The images or other third party material in this article are included in the article's Creative Commons license, unless indicated otherwise in the credit line; if the material is not included under the Creative Commons license, users will need to obtain permission from the license holder to reproduce the material. To view a copy of this license, visit http://creativecommons.org/licenses/by-nc-sa/3.0/

Supplementary Information accompanies the paper on Experimental \& Molecular Medicine website (http://www.nature.com/emm) 